\title{
Artigo \\ Desenvolvimento de um sistema embarcado para aquisição de dados em um veículo aéreo não tripulado
}

\author{
Wilknis Deyvis Silva Oliveira ${ }^{[1]}$, Rosângela Paulo de Sousa ${ }^{[2]}$, Amália Celina Santos Lacerda ${ }^{[3]}$, \\ Marcelo Roberto Bastos Guerra Vale ${ }^{[4]}$
}

[1] Universidade Federal Rural do Semi-árido; wilknisolivera@ gmail.com

${ }^{[2]}$ Universidade Federal Rural do Semi-árido;

[3] Universidade Federal Rural do Semi-árido; amalia.celina05@gmail.com

${ }^{[4]}$ Universidade Federal Rural do Semi-árido; marceloguerra@ufersa.edu.br

Recebido: $26 / 06 / 2019$;

Aceito: 21/08/2019;

Publicado: 07/10/2019.

Resumo: O seguinte trabalho apresenta um sistema embarcado desenvolvido com a finalidade de realizar a aquisição de dados de um VANT (Veículo Aéreo Não Tripulado), como exigido no edital da $20^{\circ}$ competição SAE (Sociedade de Engenheiros da Mobilidade) Brasil AeroDesign para a classe Advanced. O mesmo utilizou microcontroladores da ATMEL, ATMEGA238 E ATMEGA2560, bem como suas placas de prototipagem, um RTC (Real Time Clock) DS1307, o sensor de Efeito Hall KY-024, sensor ultrassônico HC-SR04, um medidor de pressão GY-68, o módulo MPX7002DP com Tubo de Pitot, o GPS (Global Positioning System) GY-NEO6MV2 e um acelerômetro e giroscópio GY-521. As informações obtidas foram registradas em um cartão de memória através de um adaptador para posterior análise de dados. O resultado do projeto mostrou ser capaz de realizar as tarefas de aquisição de dados de forma satisfatória, apresentando resultados consistentes para um posterior tratamento de dados e futura atualização do sistema, embora o tempo de aquisição de dados tenha se dado em uma frequência de apenas $1 \mathrm{~Hz}$. Ainda, foram realizados ensaios individuais para obtenção de dados da rotação do motor, distância por sensor ultrassônico, altitude barométrica, velocidade anemométrica e percurso por GPS.

Palavras-chave: VANTs; embarcado; AeroDesign; microcontroladores

\section{INTRODUÇÃO}

$\mathrm{A}$ facilidade de locomoção e a miniaturização dos equipamentos fornecem ao mercado de VANTs um amplo espaço de finalidades. Em suma, todas as aplicações dessas aeronaves podem ser divididas em quatro grupos: ambiental, segurança, comunicação e monitoramento [1]. Seu destaque está sempre aliado à obtenção de imagens de forma remota, podendo ser utilizadas em inspeção de linhas de transmissão, agricultura de precisão, tubulação de gás e tráfego urbano. Ainda, tais imagens já demostraram seu grande potencial para utilização em desastres naturais [2], controle de queimadas, controle de desmatamento e combate ao tráfego de drogas, contrabando e entrada de armas ilegais pela fronteira [3].

Dessa forma, para execução de um percurso seguro e eficiente, são necessários instrumentos de voo que auxiliem o piloto na observação de condições operacionais da aeronave e identificação de possíveis problemas que venham a surgir, contribuindo na tomada de decisões. Tais instrumentos podem ser definidos para condições operacionais de voo e da aeronave, ou para o comportamento da mesma, podendo fornecer informações providas pelo motor, como temperatura e rotação, nível do combustível, bússola, indicador de atitude e altitude, velocidade e GPS [4].

Considerando a importância do desenvolvimento dessas tecnologias, a SAE Brasil AeroDesign realiza anualmente uma competição onde um dos principais objetivos, é o aprendizado na área aeronáutica [5]. Esta, se dá por meio do desenvolvimento de um VANT rádio controlado, em todo seu ciclo de produção, desde a parte de projeto, passando pela construção e seus devidos testes. Assim, a competição é dividida nas categorias Micro, Regular e Advanced. Cada uma apresenta requisitos e objetivos específicos, fornecendo um determinado 
desafio de engenharia na produção da aeronave. Essas características exigidas mudam e se renovam de acordo com o avanço da edição, sendo especificadas por meio de um edital que integra um sistema de pontuação.

A $20^{a}$ edição da competição, em sua classe Advanced, apresenta três meios de pontuação voltados para aquisição de dados: Requisitos de Sistemas Embarcados; Missão de Reconhecimento; e Pontuação por Aquisição de Dados. Assim, o presente trabalho tomou como objetivo a implementação do primeiro e segundo sistema de pontuação.

O item Requisitos de Sistemas Embarcados, exige a implementação de um relógio, contado em segundos, medido em relação ao horário de Brasília, e o valor da rotação do motor em RPM (rotações por minutos). Já o item Pontuação por Aquisição de Dados, especifica os seguintes requisitos: parâmetro binário, sendo 1 para voo e 0 para solo; altitude barométrica; velocidade por sistema anemométrico; posição tridimensional por GPS; proa magnética; deflexão do profundor, aileron e leme; taxa de rolamento e arfagem; fator de carga; e atitude e inclinação lateral. Estes dados devem ser armazenados em um cartão SD em formas de colunas, identificados pelo seu respectivo parâmetro e unidade, como especificados nas seções 8.4 e 8.11.5 do edital.

Para a cumprimento de cada ponto discorrido, foram utilizados dispositivos e sensores fornecidos pela equipe PegAzuls AeroDesign da UFERSA (Universidade Federal Rural do Semiárido). Assim, ao final destas tarefas, os dados obtidos deverão passar por breve análise e verificações de cada acuracidade e efetividade, além de serem dadas as devidas conclusões para o prosseguimento de suas aplicações, aperfeiçoamentos ou suas devidas substituições.

\section{REFERENCIAL TEÓRICO}

O presente projeto aborda uma série de dispositivos, instrumentos e sensores que contribuem para o bom funcionamento e efetividade do sistema embarcado presente no VANT. Assim, esta seção abordará diferentes teorias e fundamentações de diversos trabalhos referentes a ferramentas empregadas em sistemas embarcados similares. A seguir, será discorrido a respeito das características básicas dos VANTs e suas principais variações. Também, a presente seção abordará diferentes tipos de microcontroladores e sua utilização na medição das grandezas necessárias ao trabalho proposto.

\subsection{VANTS}

Os VANTs compreendem certa variedade de modelos e aplicações que podem ser melhor assimiladas pela definição da ABA (Associação Brasileira de Aeromodelismo), onde VANT é "um veículo capaz de voar na atmosfera, fora do efeito de solo, que foi projetado ou modificado para não receber um piloto humano e que é operado por controle remoto ou autônomo" [6].

Estas aeronaves podem ser classificadas nos seguintes termos de altitude e alcance: de mão, com 600m de altitude e alcance de $2 \mathrm{~km}$; curto alcance, com 1500m de altitude e alcance de 10km; OTAN, com 3000m de altitude e alcance de 50km; dentre outras. Ainda, mais uma classificação para os VANTs difere-os em asa fixa ou rotativa. Os de asa rotativa podem possuir um motor de propulsão, como um helicóptero convencional, ou ainda, mais de um motor, sendo classificados como multirotor [7].

Para o presente projeto, as aeronaves construídas na competição da SAE Brasil AeroDesign são classificadas como de mão e são do tipo asa fixa, em um modelo mais próximo a estrutura de um avião convencional. Assim, é importante conhecer as principais partes deste tipo de VANT, visando uma melhor compreensão da problemática aqui abordada.

Um avião pode ser definido como uma aeronave que possui uma asa fixa mais pesada que o ar, sendo movido por propulsão mecânica. Estes devem conter, não obrigatoriamente, fuselagem, asas, trem de pouso, empenagem e conjunto moto-propulsor, composto por motor e hélice [8], como pode ser observado na Figura 1.

Ainda, como identificado na Figura 1, um avião possui superfícies móveis que tornam possíveis a realização de manobras em torno de seu eixo. Estas superfícies podem ser ailerons para movimento de rolamento, profundor para movimento de arfagem, e o leme para movimento de guinada [8]. Estes movimentos serão melhor abordados na seção 2.2.5. Por fim, com o objetivo de observar grandezas fundamentais para o cumprimento dos requisitos necessários para este projeto, serão utilizados microcontroladores que irão monitorar uma série de sensores acoplados a um VANT. 


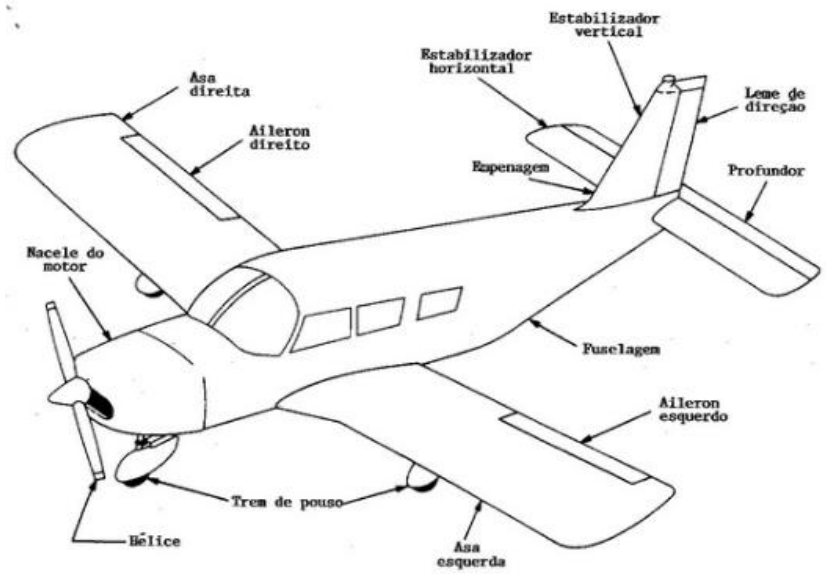

FIGURA 1. Parte de um avião. [9]

\subsection{Microcontroladores}

Um microcontrolador pode ser considerado um pequeno computador embutido em um chip, tendo as principais funções presente nos primeiros computadores domésticos: um processador, até $2 \mathrm{kB}$ de memória RAM (Random Access Memory), memória EPROM (Erasable Programmable Read Only Memory) e pinos de entradas e saídas, analógicas ou digitais [8]. Este dispositivo pode ser considerado o "cérebro" de um sistema embarcado, que comandará todas as operações de entradas e saídas, incluindo as operações lógicas e a efetiva comunicação entre os sensores e atuadores embarcados. Ao se desenvolver um projeto, é importante escolher o microcontrolador que melhor se adeque as necessidades exigidas, considerando seus recursos oferecidos, número de portas analógicas e digitais, e a relação custo-benefício.

Taglietti et al. em [10], com um projeto baseado no reuso de IPs (blocos de Propriedade Intelectual), utiliza-se de um microcontrolador RISC (Reduced Instruction Set Computer) com arquitetura Havard, o PIC (Pragrammable Interface Controller) 16F84 da Microship. Já em um sistema de aquisição de dados para um aeromodelo, Sanders e Coradini em [11], optaram por utilizar placas Arduino em comparação aos microcontroladores da Microship, devido a praticidade, facilidade e a relação custo-benefício. A placa Arduino $®$ UNO, escolhida para o sistema embarcado citado, possui um microcontrolador ATMEGA328 com 28 pinos. Destes pinos, 14 portas são digitais, das quais 6 podem ser saídas PWM (Pulse Width Modulation), com saídas de $5 \mathrm{~V}$ e fornecimento de até $40 \mathrm{~mA}$, e 6 portas são analógicas, apresentando um número de entradas/saídas suficientes para o presente trabalho. Ainda, Matos em [12], em um tutorial para sistema de aquisição de dados remoto, orienta a utilização do Arduino® UNO como unidade controladora, destacando sua memória Flash de $32 \mathrm{kB}$, sua memória EEPROM de $1 \mathrm{kB}$ e uma velocidade de clock de $16 \mathrm{MHz}$, sendo suficiente para os primeiros testes do projeto atual e a verificação do comportamento da placa em conjunto com os demais sensores.

Reforçando a utilização das placas da Atmel, Mescoloti et al. em [13], empregam o Arduino® UNO para realizar um controle de voo autônomo de um VANT, lendo dados de sensores como GPS e acelerômetro, enviando os mesmos por telemetria para uma estação de controle. O mesmo ainda indica a utilização do Arduino MEGA2560 para um sistema mais robusto, com capacidade para 54 pinos digitais, 16 portas analógicas e 256 KB de memória Flash, sendo este um número de portas mais que suficiente para a necessidade atual.

Para determinar a aplicação de um microcontrolador para a presente didática, foram analisadas as informações aqui abordadas, embasando a escolha. Assim, decidiu-se utilizar as placas Arduino em comparação aos microcontroladores da Microchip, como já orientado por Sanders e Coradini em [11].

Para desenvolvimento do presente projeto, foi utilizado a placa Arduino® Mega2560. Esta escolha se deu devido à baixa configuração de hardware oferecido pelo Arduino ${ }^{\circledR}$ UNO em relação ao presente projeto, com o programa ocupando $93 \%$ da memória dinâmica e $100 \%$ de espaço para sketch, resultando em um consequente comprometimento na execução dos códigos desenvolvidos. Assim, com a utilização do microcontrolador da Atmel ATMEGA2560, a memória dinâmica ocupou apenas 22\% de seu espaço e o sketch preenche $10 \%$ do espaço para armazenamento de programas, sendo suficiente para o bom funcionamento do sistema. 
Assim, o microcontrolador escolhido irá coordenar uma série de sensores com o objetivo de captar as grandezas necessárias para o cumprimento da proposta de trabalho. Por consequente, nas seguintes subseções será discorrido a respeito do emprego de sensores de efeito Hall para determinar a rotação de motores, da aplicação de sensores ultrassônicos para curta distância e da utilização de sensores de pressão atmosférica para determinar a altitude barométrica. Ainda, será verificado o emprego do Tubo de Pitot para definição da velocidade anemométrica de uma aeronave, aplicações de GPS, utilização dos Ângulos de Euler em conjunto com giroscópios, e a determinação dos ângulos de servomotores para verificação do ângulo das superfícies móveis de VANTs.

\subsubsection{Rotação do motor}

Utilizando microcontroladores com vista a medição da rotação do motor em RPM, é possível receber um pulso de onda quadrada, identificado a cada volta da hélice do VANT. A diferença de tempo obtida entre cada leitura pode ser medida pelo próprio microcontrolador e assim calculado o valor final em RPM. Para leitura desses pulsos, Rana, Sahabuddin e Mondol em [14], utilizam a propriedade dos fototransistores. Quando uma luz IR (Radiação Infravermelha) incide sobre um fototransistor, este retorna o valor lógico high, detectando o pulso, voltando para o valor low, quando fora de contato com a luz. Porém, a frequência da luz ambiente interfere significativamente nos resultados obtidos. Assim, uma forma de medição alternativa a esta aplica o Efeito Hall.

O sensor de efeito hall é largamente utilizado devido ao seu tamanho reduzido, altas frequências de leituras e longa vida útil. Tal efeito se dá pela passagem de corrente elétrica (I) através de uma placa dielétrica, que na presença de um campo magnético (B) produz uma tensão de efeito hall (Vhall). Em outras palavras, os elétrons passam de um lado da placa para o outro quando aplicada uma diferença de potencial [15]. Este efeito pode ser melhor visualizado através da Figura 2.

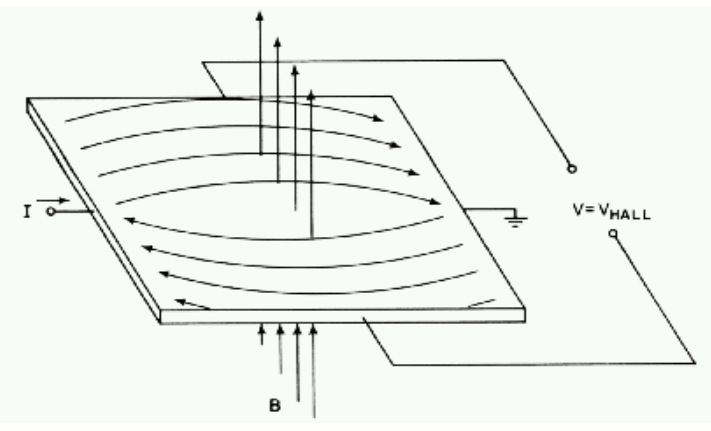

FIGURA 2. Efeito Hall. [16]

Alguns notáveis projetos já utilizaram sensores de efeito hall para desenvolvimento de tacômetros. Tisaj em [15], aplicou o sensor da Freetronics AH180 que apresenta um clock de $125 \mu$ s. Este mostrou resultados satisfatórios na leitura da frequência de rotação. Jia et al. em [17], também utilizam sensores de efeito hall para a mesma aplicação, mas desta vez em conjunto com detectores magnéticos e um circuito de filtro RC (ResistorCapacitor), apresentando resultados mais precisos. Ainda, Sanders e Coradini em [11], integraram o sensor A3144 a roda de um aeromodelo para medir a velocidade da aeronave em solo, mostrando uma diferente aplicação para esta tecnologia.

Assim, considerando a eficácia dos trabalhos que utilizam sensores de efeito Hall aqui abordados, para este projeto, foi utilizado o módulo KY-024. Este consegue realizar leituras consumindo uma corrente de $4 \mathrm{~mA}$ com alimentação de 5V, sendo suficiente para sua integração a placa Arduino® MEGA2560. Ainda, o mesmo possui um sensor linear de efeito Hall SS49E embutido para a aplicação da teoria discorrida. 


\subsubsection{Sensores ultrassônicos}

Além de conhecer a rotação do motor, identificando possíveis falha durante o percurso, saber o devido momento em que a aeronave levanta voo é essencial para realizar uma análise correta dos dados obtidos ou transmitidos. Esta função pode ser bem estabelecida pela detecção de proximidade por sensores ópticos, bastante utilizados na indústria. Porém, objetivando uma melhor análise do momento de voo, os sensores ultrassônicos se demonstraram mais interessante para o presente caso, devido a capacidade de definição da distância.

Em um estudo para aquisição do erro máximo obtido pelo sensor ultrassônico HC - SR04, Nakatani, Guimarães e Neto em [18], chegaram a um valor máximo de -9,35mm para uma distância de 80mm, apresentando resultados mais que suficientes, já que o presente trabalho não necessita de uma precisão milimétrica. Sanders e Coradini em [11], também aplicaram a utilização deste sensor para definir o momento da decolagem de um aeromodelo, fixando o mesmo a baixo da fuselagem. O dispositivo em questão, é composto por um transmissor ultrassônico, um receptor e um circuito de controle, fornecendo uma medição na faixa de $0,02 \mathrm{~m}$ à $4 \mathrm{~m}$. Seu princípio de funcionamento se dá pelo envio de 8 ciclos de pulsos em uma frequência de $40 \mathrm{kHz}$, com ângulo de $15^{\circ}$, pelo pino trigger, ao receber uma tensão de $5 \mathrm{~V}$ por um período de $5 \mu \mathrm{s}$. Assim, o tempo entre o envio do sinal e o seu recebimento pelo pino echo é multiplicado pela velocidade do som e dividido por 2 (tempo de ida e volta), obtendo a distância em centímetros [19]. Dessa forma, o dispositivo HCSR04 foi escolhido para identificar quando o avião ultrapassa 1,5m em relação ao solo, margem de cumprimento dentro da faixa de medição oferecida pelo dispositivo.

\subsubsection{Sistema anemométrico}

Verificado o início do voo através do sensor ultrassônico, os dados referentes a velocidade do VANT podem ser melhor analisados. Assim, para aquisição destes dados, um sistema de velocidade anemométrico deve obter a leitura da velocidade aerodinâmica, correlacionando a pressão do tubo de Pitot $\left(P_{t}\right)$ e a pressão atmosférica no local da leitura, adquirida pela tomada estática $\left(P_{e}\right)$ (ver Figura 3).

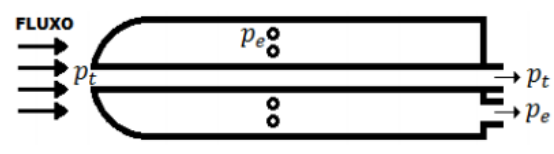

FIGURA 3. Tubo de Pitot.. [19]

Este cálculo de velocidade se dá pelo princípio de escoamento de fluidos de Bernoulli, evidenciado na Equação 1, onde relaciona a velocidade aerodinâmica $(V)$, pressão estática $\left(P_{\text {estática }}\right)$, pressão dinâmica $\left(P_{\text {dinâmica }}\right)$ e densidade do fluido $(\gamma)$. Para a prática desta teoria, é necessário a utilização de um sensor de pressão diferencial que irá medir a diferença de pressão entre as duas tomadas, calculando a velocidade aerodinâmica da aeronave [20].

$$
\mathrm{V}=\sqrt{2 *\left(\mathrm{P} \_ \text {estática-P_dinâmica }\right) \gamma}
$$

Em um estudo para sensoriamento em aeromodelos, Sousa e Margi em [20], fazem uso do sensor modelo 850S da VectusImportatum, desenvolvendo um ensaio para determinação da curva entre pressão de entrada e saída. Os resultados evidenciaram uma medição máxima de $100 \mathrm{~Pa}$, relativa a uma velocidade de $13 \mathrm{~m} / \mathrm{s}$, o que apresenta ser insuficiente, já que serão necessárias leituras à um máximo de 22m/s. Já Silva em [21], optou pela escolha de um tubo de Pitot para aeromodelo, o qual possui quatro entradas laterais para pressão estática e uma em sua ponta para pressão de estagnação. Para determinar a diferença de pressão entre as tomadas, o mesmo acrescentou o sensor MPX7002DP da Freescale, que possui uma faixa de $-2 \mathrm{kPa}$ a $2 \mathrm{kPa}$ e uma sensibilidade de $1 \mathrm{~V} / \mathrm{kPa}$. Porém, é afirmado que o sensor apresenta um erro de até $6,5 \%$, correspondente a um valor de $250 \mathrm{~Pa}$. Porém, esta faixa de leitura já compreende o necessário para o presente projeto.

Além de também incorporar o sensor MPX7002DP, Sanders e Coradini em [11], adiciona o sensor DHT11, para determinação da umidade relativa do ar, e o BMP180, que além de obter a pressão atmosférica de forma mais precisa, consegue informar a temperatura e a altitude barométrica, também pelo princípio de Bernoulli. Assim, com este conjunto de sensores é possível determinar a variação da pressão com relação a 
altitude e a variação da densidade do ar com maior precisão. Ainda, Serbezov, Dimitrov e Rangelov em [22], apresentam o sensor para medição de pressão e temperatura BMP-183, e o tubo de Pitot HD350, conectado via serial USB a um computador. Porém, este último torna sua aplicação para o presente projeto inviável, devido a necessidade de conexão USB.

Assim como aplicado por Sanders e Coradini em [11], foi empregado um tubo de Pitot para aeromodelo no presente trabalho, em conjunto com um módulo MPX7002DP para cálculo da velocidade aerodinâmica, e um módulo BMP180 para obtenção de dados relativo a altitude barométrica.

\subsubsection{Localização GPS}

Além de obter as informações anteriormente comentadas, é necessário conhecer o percurso realizado pelo VANT, correlacionando os demais dados a posição da aeronave. Assim, a posição geográfica do VANT pode ser obtida através de um GPS (Global Positioning System). Largamente comercializado com aplicações em diversas áreas tecnológicas, o protocolo GPS encontra-se em uma ampla gama de aparelhos eletrônicos com inúmeras finalidades. Este pode ser encontrado em computadores, celulares, veículos automotivos, aeronaves e dentre outras. Tal recurso é capaz de receber informações da NMEA (National Marine Electronics Association), por meio de 24 satélites geoestacionários, sendo necessário até 3 satélites para obter a localização e mais um para determinação da distância em relação ao nível do mar [23].

Em uma aplicação para controle de um quadricóptero, Silva e Morais em [2], apresentam o GPS EM-406A da GlobalSat, com um consumo de apenas $25 \mathrm{~mA}$, uma precisão de $0,1 \mathrm{~m} / \mathrm{s}, 5 \mathrm{~m}$ para posição e sincronização de $1 \mu$ s. Enquanto Pebrianti et al. em [24], também em aplicações para quadricópteros, se faz de um sensor GYNEO6MV2 da linha Ublox NEO-6M, com precisão de 2,5m para localização e reposta de satélite em 1 segundo [25].

Assim, para determinar a posição cartográfica, medida em metros e contada a partir do início do voo, utilizou-se o GPS GY-NEO6MV2, devido a sua melhor precisão de posição se comparado ao EM-406A. Por fim, o GPS retorna valores de latitude e longitude, assim, se faz necessário a conversão para o sistema de coordenadas UTM (Universal Tranversa de Mercator), o qual irá fornecer dados em metros, definidos cartograficamente para diferentes regiões do globo.

\subsection{5. Ângulos de Euler e o Fator de Carga}

Apenas a verificação da posição geográfica da aeronave não é suficiente para definir a movimentação da mesma ao longo de um percurso. Menores manobras, não são detectáveis por GPS e podem ser verificadas através de sensores de medição inercial, como giroscópios. Ao se utilizar de um giroscópio, é necessário a definição de um sistema para orientação de objetos no espaço, sendo escolhido para o presente projeto, os Ângulos de Euler. Estes, como melhor observado na Figura 4, são compostos pelos ângulos de rolamento, com inclinação em torno do eixo $\mathrm{X}$, de arfagem, com inclinação em torno do eixo $\mathrm{Y}$, e de guinada, com inclinação em torno do eixo Z [21].

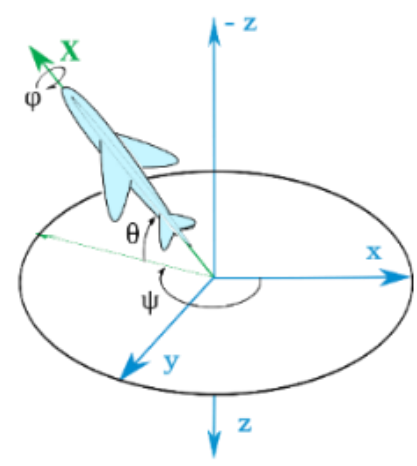

FIGURA 4. Ângulos de Euler. [26]

Tal sistema de orientação possui uma falha durante uma manobra de Gimbal Lock, a qual se dá quando o ângulo de arfagem chega aos $90^{\circ}$, fazendo os movimentos de rolamento e guinada transitarem no mesmo eixo 
[21]. Porém, essa falha é desprezível para a aplicação em questão, pois os VANTs desenvolvidos para a Competição SAE Brasil AeroDesign são aeronaves de cargas, não chegando a desenvolver manobras bruscas.

Dessa forma, para obtenção destes ângulos, se faz necessário a utilização dos giroscópios, os quais possuem a capacidade de mensurar a velocidade angular de um objeto. Estes tornam possível estimar o ângulo desejado, através de uma multiplicação entre esta velocidade angular, em um determinado eixo, e o tempo que essa velocidade exerceu, informado pelo próprio microcontrolador. Porém, esse processo induz ao erro drift, que está associado a fabricação do componente e a velocidade da leitura dos dados [21].

Adicionado para a determinação de tais ângulos, Silva e Morais em [2], utilizam um módulo IMU (Inertial Measurement Unit) que integra acelerômetro, giroscópio e magnetômetro, resultando em nove graus de liberdade. Porém o mesmo não informa a referência do sensor e fabricante. Já Silva em [21], após uma análise de mercado, escolhe o módulo GY-87, o qual possui um módulo MPU6050 com processador interno, acelerômetro e giroscópio. Ainda, os acelerômetros inclusos nos módulos citados, são capazes de obter dados para fator de carga, no eixo $\mathrm{Z}$, como exigido no edital da competição.

Para a presente problemática, as taxas de rolamento e arfagem, os ângulos de atitude e inclinação lateral, a proa magnética, bem como o fator de carga, foram requisitados pelo sensor, acelerômetro e giroscópio, GY521, através do módulo MPU6050, em uma alternativa ao GY-87 utilizado por Silva em [21]. Como o endereço referente ao MPU6050, 0x68, é o mesmo endereço do RTC DS 1307, utilizado no presente projeto, é necessário ligar o pino AD0 do GY-521 a uma tensão de 3.3V, alterando o endereço para 0x69.

Visando definir os ângulos de proa magnética, atitude e inclinação lateral, foram obtidas as devidas taxas de velocidade angular para seus respectivos ângulos. Essas taxas são multiplicadas pelo tempo, contado pelo próprio microcontrolador, resultando em um ângulo que será somado ao anterior. Dessa forma, o atraso de processamento impostos pelos demais dispositivos do sistema embarcado, induz o erro drift. Para minimizar esse erro, foi necessário a utilização de um microcontrolador dedicado ao sensor GY-68, o ATMEGA238, incluído na placa Arduino® UNO. A comunicação deste último com o Arduino® MEGA2560 se faz pela comunicação serial I2C (Inter-Integrated Circuit), em um protocolo mestre/escravo.

\subsubsection{Servomotores}

Os movimentos anteriormente descritos pelos ângulos de Euler, são provocados pelo acionamento de superfícies móveis presentes nos VANTs. Assim, comumente utilizados em aeromodelos, os servomotores são atuadores que tem como função o acionamento dessas superfícies. Estes possuem um potenciômetro acoplado ao seu eixo de rotação que é acionado por um conjunto de engrenagens de redução. Tais dispositivos possuem um circuito para entrada e saída de 3 fios: um fio vermelho para uma tensão de 5V, um fio preto para o GND (Ground) e um fio amarelo, ou branco, para um sinal PWM, que faz o controle de posicionamento do eixo através do potenciômetro [22].

O sinal PWM possui dois parâmetros que os definem, a frequência e a razão do ciclo. Esta frequência varia de acordo com o modelo do servomotor, porém a largura do pulso é quem irá definir a posição do eixo. Segundo Filardi em [27], testes com o modelo Futaba S3003 apresentam um ângulo de $-90^{\circ}$ para uma largura de pulso de $0,1 \mathrm{~ms}$ e um ângulo de $90^{\circ}$ para um valor de $2,3 \mathrm{~ms}$. Dessa forma, este processo foi aplicado no presente trabalho para três atuadores, referentes ao profundor, aileron e leme.

\section{Metodologia}

Os testes para o circuito desenvolvido se deram apenas de forma estática, devido a indisponibilidade de tempo para execução de aquisições em uma aeronave. Assim, é apresentado uma metodologia onde será estabelecido um teste com o sistema completo de forma estática, enquanto aqueles sensores que necessitam de uma devida variação de dados de forma dinâmica, terão ensaios individuais para comprovar a devida efetividade da obtenção de dados.

\subsection{Sistema completo para teste estático}

O sistema embarcado desenvolvido, compreende um circuito que contempla todos os sensores e dispositivos escolhidos ao longo da fundamentação teórica, como pode ser observado na Figura 5. Os dados obtidos por cada sensor foram armazenados em um cartão SD (Secure Digital card) através do módulo Card Adapter. Ainda, objetivando uma melhor manipulação do sistema, foi adicionado mais dois componentes ao circuito: um botão e um led. O primeiro terá a função de arrancar a inicialização dos sensores e, 
consequentemente, a efetiva aquisição dos dados. Já o segundo, irá piscar a uma frequência de 1s durante a aquisição de dados, ou ainda, caso ocorra um erro na comunicação com os sensores, irá piscar a um intervalo de $50 \mathrm{~ms}$.

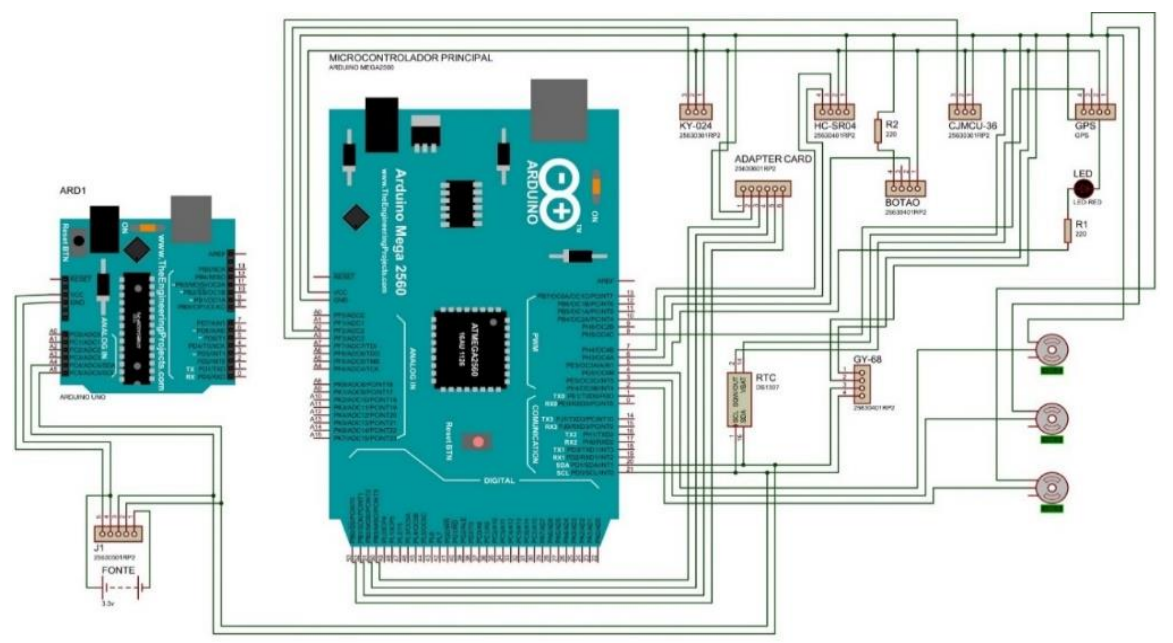

FIGURA 5. Circuito completo. (Autoria própria)

Observa-se que os sensores KY-024, de efeito Hall para rotação do motor, e o sensor CJMCU-36 com módulo MPX7002DP para cálculo da velocidade aerodinâmica, estão conectados a saídas analógicas. O protocolo I2C foi empregado, utilizando as conexões SDA (Serial Data) e SCL (Serial Clock) nos sensores DS1307 para cálculo de tempo, GY-68 para obtenção da altitude, GY-521 para ângulos de Euler e fator de carga, e no Arduino ${ }^{\circledR}$ UNO, para controle do sensor acelerômetro e giroscópio GY-521. Já o protocolo UART (Universal Synchronous-Asynchronous Receiver) foi utilizado pelo módulo Card Adapter. Por fim, os demais sensores estão conectados a portas digitais.

O código desenvolvido pode ser observado através do fluxograma apresentado na Figura 6. O programa, inicia-se em duas frentes, um fluxo para o Arduino ${ }^{\circledR}$ UNO, que controlará o sensor GY-521 (ver Figura 7 (b)) e outro para o Arduino ${ }^{\circledR}$ MEGA2560 (ver Figura 7 (a)) que comandará os demais sensores e dispositivos presentes no circuito, e receberá os dados do Arduino ${ }^{\circledR}$ UNO.
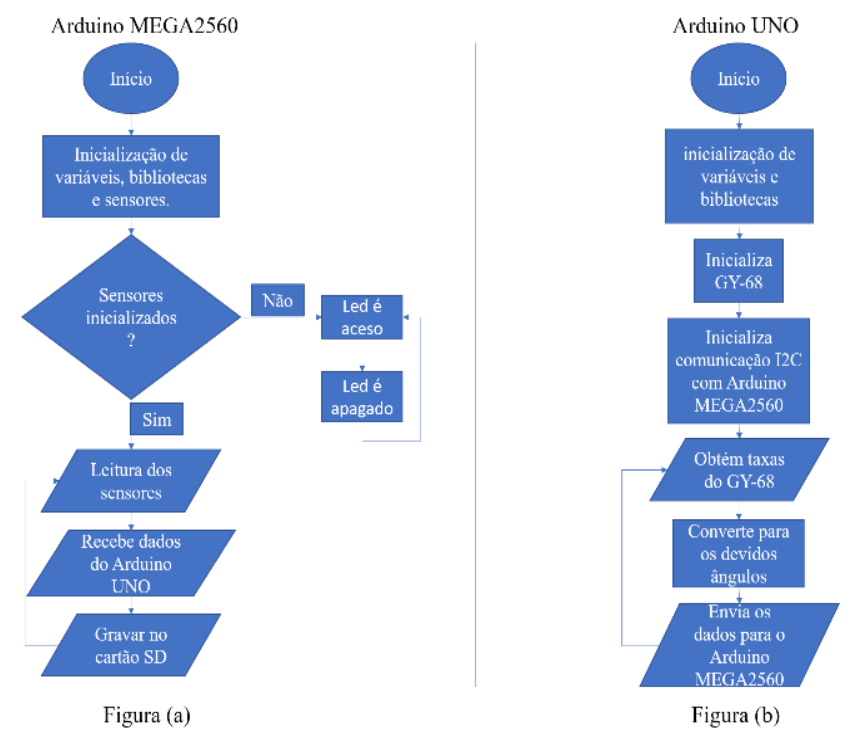

FIGURA 6. Fluxograma do código para o sistema embarcado. (Autoria própria)

O programa executa ações para cada dispositivo de maneira semelhante. Obtém os dados, realiza um tratamento das informações, armazena no cartão SD e por fim, volta para o início do loop ao final de todas as 
aquisições. Verifica-se também um loop induzido caso algum sensor não seja inicializado, fazendo o led piscar, dando a abertura para a correção de alguma possível inconformidade no circuito. Ainda, analisando o fluxograma, constata-se a transmissão dos dados do Arduino ${ }^{\circledR}$ UNO para o Arduino ${ }^{\circledR}$ MEGA2560 ao final do loop do microcontrolador escravo.

\subsection{Ensaios individuais}

Ao todo, os ensaios individuais compreenderam cinco testes. O primeiro utilizou o sensor de efeito Hall KY-024 para medição das rotações do motor em RPM. Para isso, foi empregado um motor Turnigy D2826-10 1400kv Brushless, onde foi fixado dois ímãs de neodímio ao espaçamento da hélice para contato do campo magnético com o sensor de efeito Hall. Assim, os dados foram obtidos para amostras aleatórias entre uma faixa de 1440RPM a 4560RPM, os quais foram comparados com valores fornecidos pelo tacômetro digital comercial Turnigy Micro Tacho.

O segundo ensaio foi realizado com o sensor ultrassônico HC-SR04, o qual tem a finalidade de definir o momento de início e final de voo. Para tanto, foi comparado os dados fornecidos pelo sensor com valores reais obtidos através de uma trena, variando as amostras em uma faixa de $0,05 \mathrm{~m}$ a $2,5 \mathrm{~m}$, respeitando o ângulo de $15^{\circ}$ com relação a uma superfície plana.

O terceiro ensaio empregou o sensor de pressão GY-68 para obtenção da altitude barométrica em metros. De forma semelhante ao ensaio anterior, foi utilizada uma trena para realizar comparativos com os dados obtidos do dispositivo em questão, variando uma faixa de $0,5 \mathrm{~m}$ a $5 \mathrm{~m}$.

O quarto ensaio analisou os dados do módulo MPX7002DP com um tubo de Pitot para aeromodelo, visando a obtenção da velocidade aerodinâmica. Devido a indisponibilidade de um túnel de vento para realização deste ensaio, foi utilizado um carro para obtenção da velocidade real pelo velocímetro. Enquanto o sistema anemométrico permanecia fora do veículo para contato com o escoamento do fluido, os dados foram obtidos em uma faixa de amostragem de $5,55 \mathrm{~m} / \mathrm{s}(20 \mathrm{~km} / \mathrm{h})$ a $22,22 \mathrm{~m} / \mathrm{s}(80 \mathrm{~km} / \mathrm{h})$.

Por fim, o último ensaio empregou o sensor GY-NEO6MV2 para obtenção do percurso realizado. Para este teste, um percurso aleatório foi traçado obtendo dados de forma continuada, onde as coordenadas requisitadas devem traçar um mapa com eixos em metros em um gráfico de dispersão.

\section{RESULTADOS E DISCUSSÕES}

Os dados aqui apresentados foram divididos entre resultados para teste estático, com sistema embarcado completo, e resultados para os testes dos sensores individuais. Quando um dado do sistema completo não for suficiente para discorrimento de seus valores, os resultados para testes individuas assim o fará, exceto pelos dados provenientes do sensor GY-521 e obtenção dos ângulos dos servomotores, que não apresentam metodologia para teste individual.

\subsection{Teste estático}

O sistema para aquisição de dados produzido pode ser observado na Figura 7 e parte de seus dados resultantes do teste estático, extraídos do cartão SD em formato txt, podem ser verificados na Figura 8. Analisando uma parcela dos dados provenientes do arquivo txt, verifica-se na primeira coluna, com o parâmetro Tempo (unidade em segundos), obtidos pelo RTC DS1307, uma frequência de aquisição de dados de 1 segundo $(1 \mathrm{~Hz})$ muito a baixo da frequência de $100 \mathrm{~Hz}$ desejada. O parâmetro RPM_1 (unidade em RPM) não apresentou resultados, pois não foram obtidos dados quanto a rotação do motor para este ensaio, sendo discorrido sobre o mesmo na seção 4.2 . 


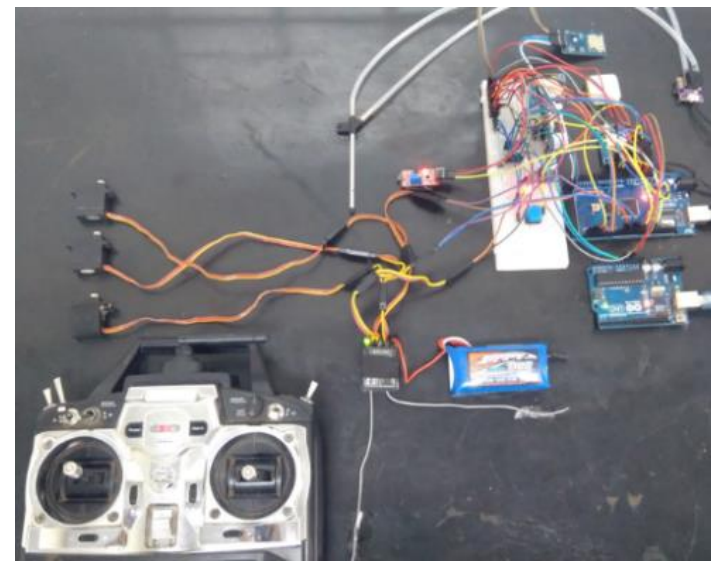

FIGURA 7. Sistema finalizado. (Autoria própria)

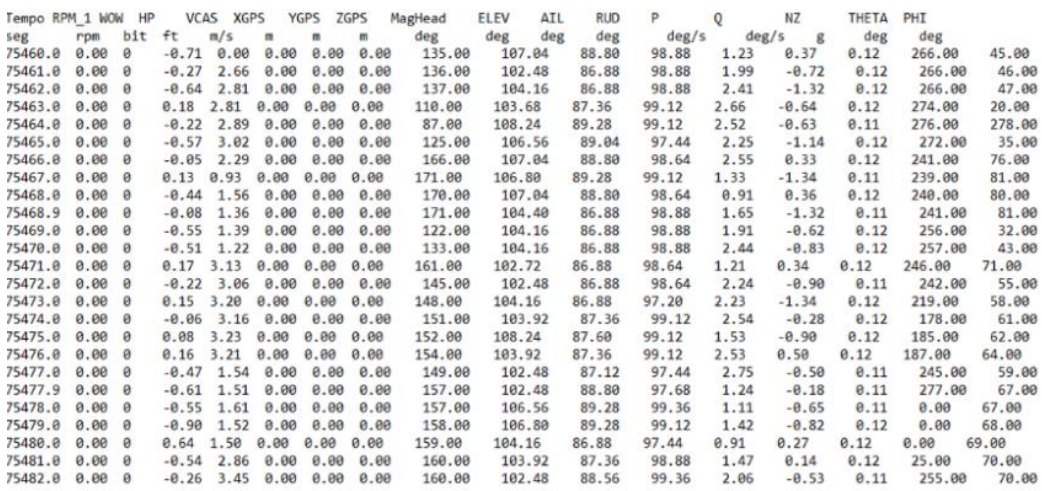

FIGURA 8. Dados do teste estático. (Autoria própria)

Verifica-se nos dados do parâmetro HP (unidade em ft), informados pelo sensor GY-68, uma variação de altitude barométrica com valores negativos, evidenciando uma falha. Isso não pode ocorrer, já que a altura 0.00ft inicia-se em solo. O parâmetro VCAS (unidade em $\mathrm{m} / \mathrm{s}$ ), obtido pelo sistema anemométrico, apresenta uma variação na velocidade, apontando mais uma falha, pois para o teste estático devia ser obtido velocidade nula.

Os parâmetros XGPS, YGPS e ZGPS (unidades em metros), referentes ao módulo GPS GY-NEO6MV2, apresenta resultados coerentes para o teste estático. Quanto aos demais dados, verifica-se uma efetiva comunicação com os sensores e atuadores, embora a acuracidade dos dados não possa ser determinada.

\subsection{Testes individuais}

O ensaio com o sensor KY-024 para obtenção dos valores de rotação do motor em RPM, evidenciou um aumento crescente na diferença entre os valores do sensor e os valores reais obtidos pelo tacômetro comercial, como pode ser observado na Figura 9.

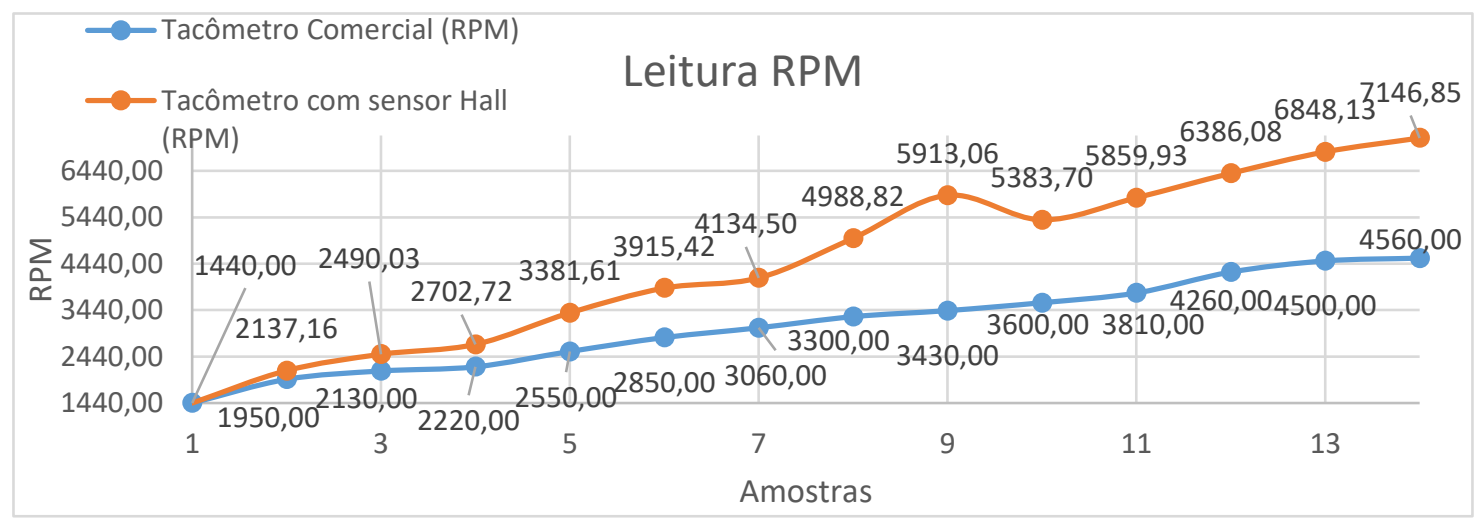

FIGURA 9. Ensaio com KY-024. (Autoria própria) 
Porém, esta diferença de resultados entre os tacômetros pode ser ajustada pela Equação 2, com uma precisão em cerca de $98 \%$, onde $(y)$ é a aproximação para um valor mais próximo ao lido pelo tacômetro, e (x) é o valor lido pelo sensor de efeito Hall.

$$
y=9,0593 * x^{0,6968}
$$

Ainda, é importante atentar que para cada amostra identificada pelo tacômetro comercial, foram obtidas um mínimo de 70 amostras pelo sensor de efeito Hall. Isso foi necessário para se obter o desvio padrão entre os dados coletados e uma consequente média, obtendo um valor mais conciso para comparação com o tacômetro comercial, já mostrado na Figura 9. Os dados obtidos para cada amostra apresentaram um desvio padrão máximo de 820,93 RPM entre os dados coletados pelo sensor de efeito Hall, quando a rotação identificada pelo tacômetro comercial chega a 4560 RPM (ver Figura 10). Ainda, a linha de tendência, com uma expressão polinomial, evidencia uma aproximação ao comportamento da variação do desvio padrão, em relação aos dados obtidos com o aumento da velocidade de rotação do motor.

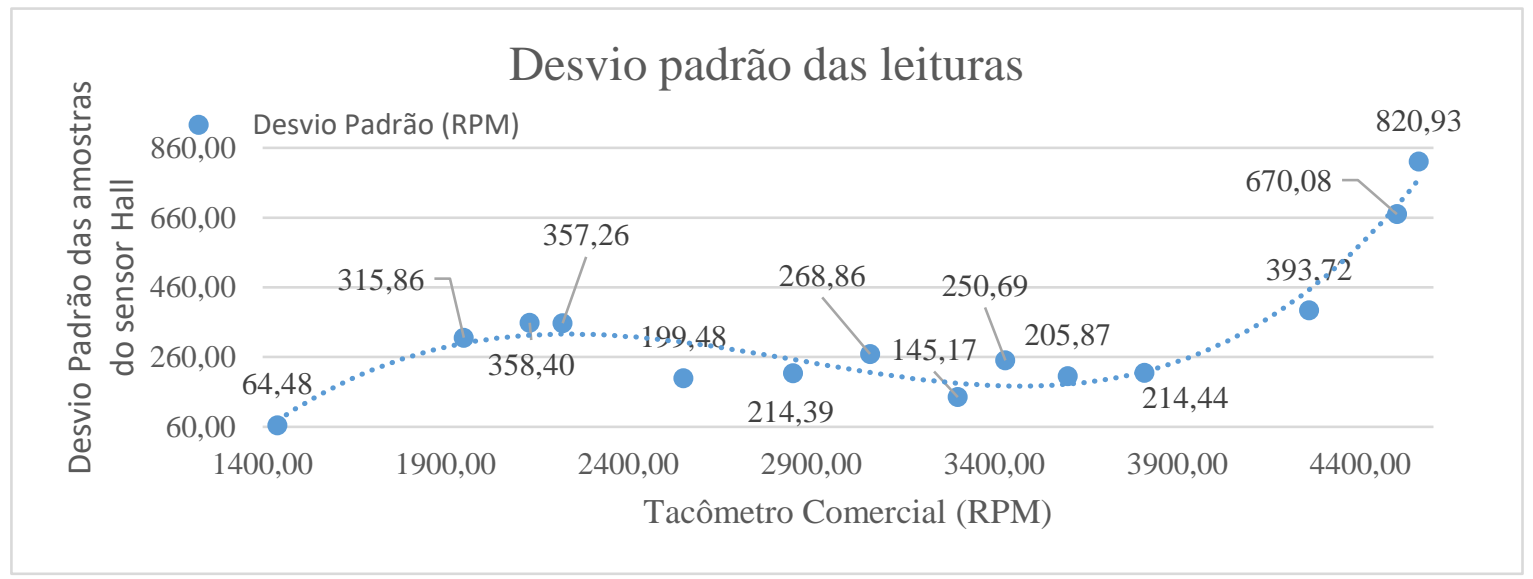

FIGURA 10. Desvio Padrão para KY-024. (Autoria própria)

Já o sensor HC-SR04, dedicado a determinação do início e final de voo, apresentou dados mais concisos, como pode ser observado através da Figura 11, a qual evidencia resultados sempre dentro do esperado. Considerando que a metodologia empregada para obtenção dos dados não considera uma exatidão milimétrica, a variação de valores apresentada no gráfico pode ser ignorada.

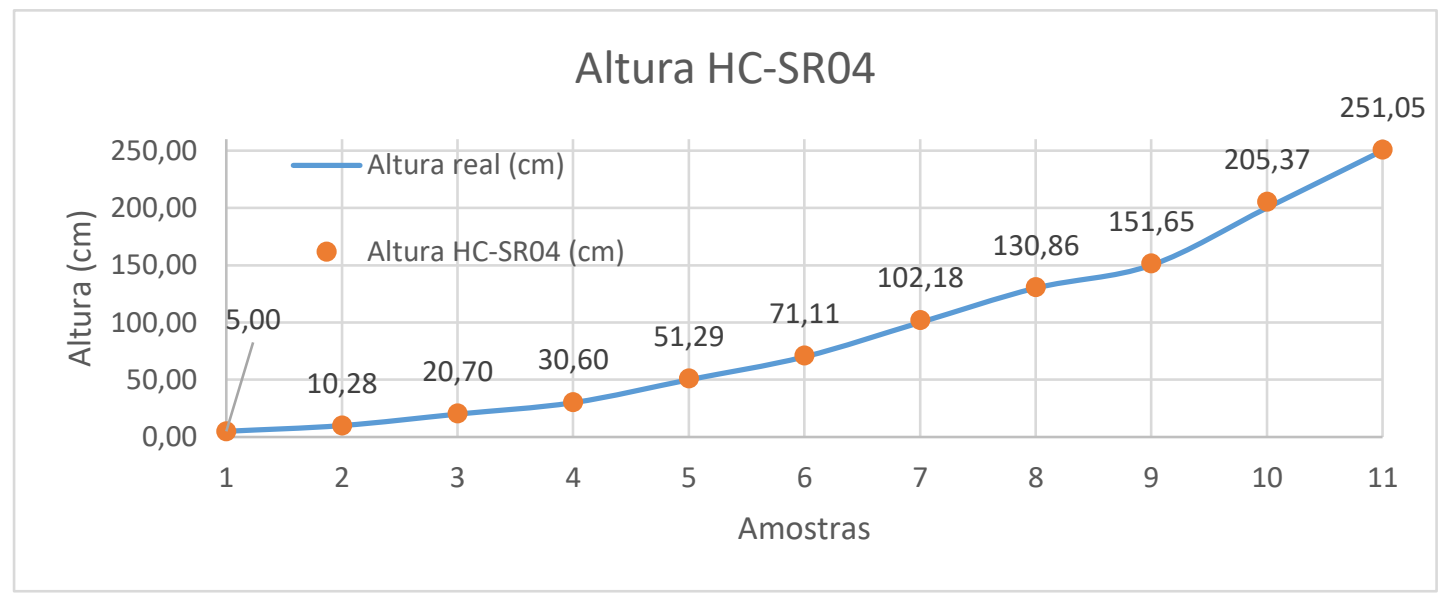

FIGURA 11. Ensaio com HC-SR04. (Autoria própria)

Testes com o sensor GY-68 para a determinação da altitude barométrica, geraram o gráfico apresentado na Figura 12. Para estes dados, a variação máxima observada é de apenas 1,5m, em uma altura real de 4,5m. 
Esta diferença pode ser considerada irrisória para uma faixa de altitude em até $600 \mathrm{~m}$, máximo para uma aeronave classificada como de mão.

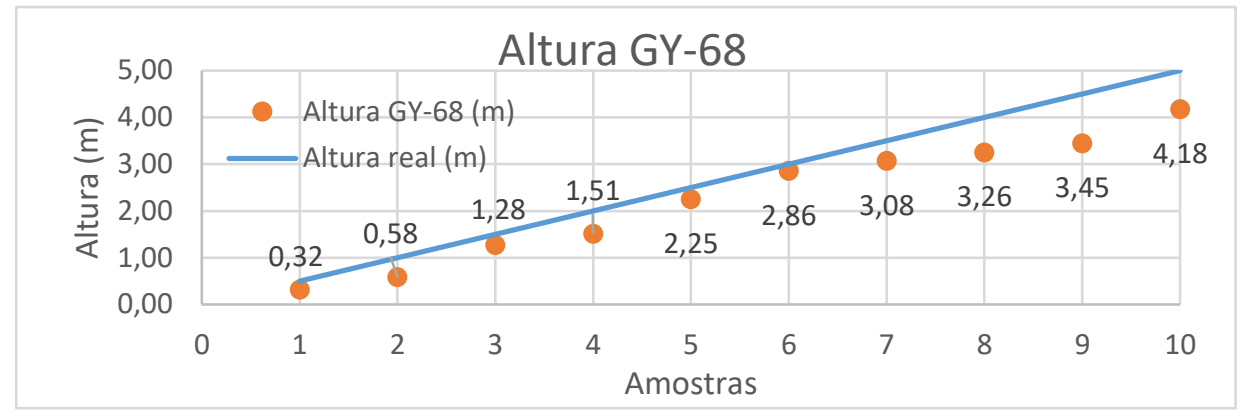

FIGURA 12. Ensaio com GY-68. (Autoria própria)

Ainda, é possível filtrar os resultados apresentados na Figura 12 para uma altitude mais próxima do valor real, através da Equação 3, com uma precisão próxima a 97\%. Esta equação relaciona o valor obtido pelo sensor $(x)$ a um valor aproximado ao real $(y)$.

$$
y=1,1508 * x+0,1291
$$

Após adquirir os dados através do ensaio do módulo MPX7002DP com o tubo de Pitot para a determinação da velocidade aerodinâmica, foi montada a Figura 13, evidenciando os dados consideráveis para a aplicação do devido dispositivo. Nesta, é possível verificar uma diferença máxima de $1,27 \mathrm{~m} / \mathrm{s}$ entre a velocidade real, lida no velocímetro e a velocidade obtida pelo tubo de Pitot, para uma leitura ideal de $22,22 \mathrm{~m} / \mathrm{s}$. Ainda, é possível afirmar que a metodologia utilizada não fornece uma precisão necessária para considerar valores a baixo de $2 \mathrm{~m} / \mathrm{s}$. Porém, é importante ressaltar que o desvio padrão médio para as amostras de dados percorrem um valor de $0,71 \mathrm{~m} / \mathrm{s}$, ainda que este seja um número considerável para aplicação em VANTs.

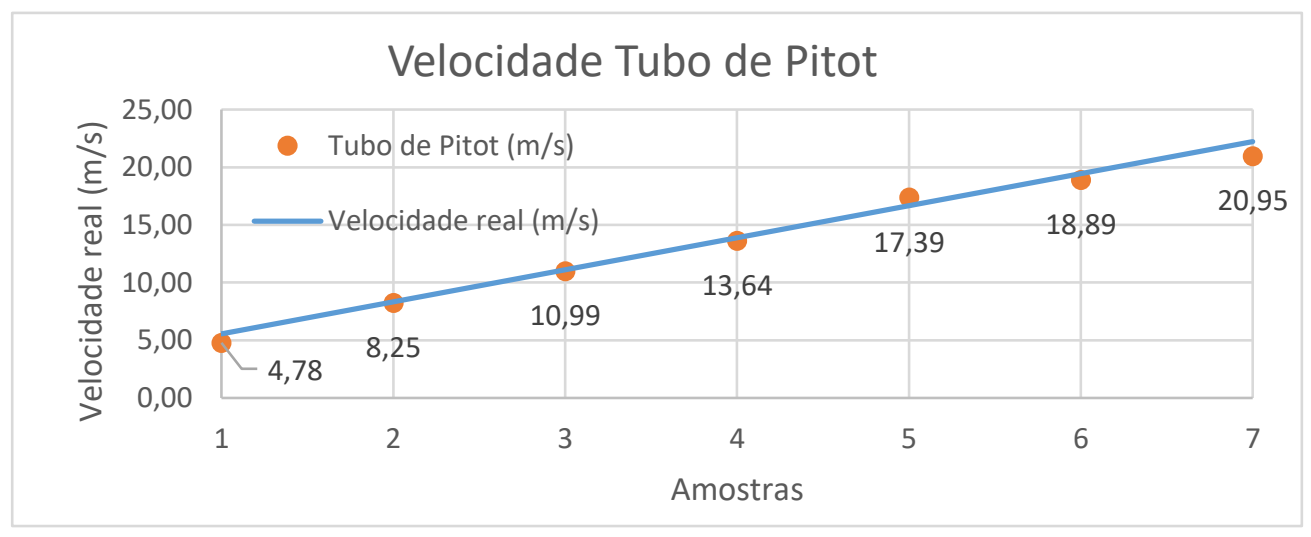

FIGURA 13. Ensaio com MPX7002DP. (Autoria própria)

O último ensaio obteve as coordenadas cartográficas de um trajeto percorrido com o auxílio do GPS GYNEO6MV2, convertendo dados de latitude e longitude para um sistema cartográfico em metros, como exibido na Figura 14. É importante salientar que os dados para altitude não foram inclusos, já que o trajeto foi realizado em solo, sendo insignificante a variação no eixo z. 


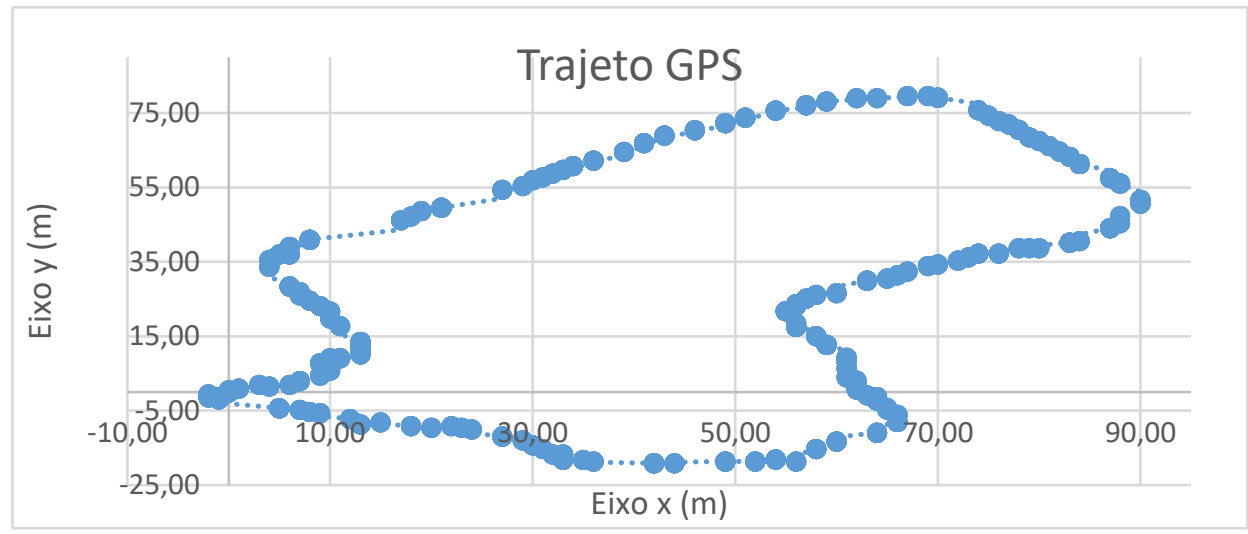

FIGURA 14. Trajeto por GPS. (Autoria própria)

Por fim, a título informativo, o percurso para aquisição das coordenadas por GPS foi realizado de forma aleatória dentro do campus Leste da UFERSA/Mossoró.

\section{CONCLUSÃO}

É importante salientar que o presente estudo não tem como objetivo a obtenção de dados precisos dos sensores aqui utilizados e sim a devida integração dos mesmos a um sistema de aquisição de dados para futuro aprimoramento. Assim, é possível concluir que o sistema embarcado desenvolvido atendeu aos requisitos para obtenção de dados, como consta nas seções 8.4 e 8.11.5 do edital, embora o mesmo não seja capaz de obter dados a uma frequência de $100 \mathrm{~Hz}$, tendo seus melhores resultados uma frequência de aquisição de $1 \mathrm{~Hz}$. Assim, se faz necessário um mapeamento de utilização de tempo por cada dispositivo para definir quais podem ser otimizados, visando a redução do tempo total de aquisição.

Os dados obtidos para rotação do motor em RPM pelo ensaio do sensor de efeito Hall KY-024, apresentam uma enorme diferença de dados entre o tacômetro comercial utilizado e o desenvolvido. Embora estes possam ser melhores ajustados por uma equação como comentado anteriormente, é necessário definir o problema que resulta o aumento exponencial dos valores de RPM por parte do sensor de efeito Hall. Ainda, pode-se destacar o alto desvio padrão apresentado de acordo com o aumento da velocidade de rotação da hélice, podendo prever através da curva presente na Figura 9, resultados inutilizáveis em valores acima de 7500 RPM.

Já os sensores HC-SR04 e GY-68, se mostraram confiáveis para a suas devidas aplicações, considerando valores de ângulo de curva e desvio padrão aceitáveis. Da mesma forma, os dados obtidos para o ensaio dedicado a medição de velocidade por tubo de Pitot, apresentaram resultados confiáveis e lineares, com um desvio padrão baixo e resultados sempre próximos ao esperado.

Quantos aos dados disponibilizados pelo GPS, não foi possível determinar a exatidão das informações adquiridas, embora a tarefa de aquisição de dados do sensor e formação de um trajeto, tenham sido realizadas.

A utilização do microcontrolador ATMEGA238, dedicado ao módulo GY-521, cumpriu com sua finalidade, obtendo as informações do sensor de forma continuada, e minimizando o erro Drift associado, apesar de não ter sido possível determinar a quantidade de erro ainda existente, devido à ausência de uma metodologia com essa finalidade neste trabalho.

Embora a aquisição da posição do eixo do servomotor tenha obtido os resultados em ângulos, estes dados não correspondem ao exato grau de liberdade da superfície móvel, sendo necessário uma equação que correlacione a posição do eixo dos atuadores ao exato posicionamento do profundor, aileron e leme da aeronave.

Para futuros trabalhos, é necessário aprimorar o projeto para uma aquisição de dados para frequências acima de $100 \mathrm{~Hz}$, realizar ensaios dedicados a acuracidade do sensor acelerômetro e giroscópio GY-521 e ensaios para determinação da acuracidade dos ângulos dos servomotores, bem como sua correlação com os ângulos das superfícies móveis de um VANT. Ainda, pode-se implantar um sistema de telemetria para envio remoto de dados e imagens, bem como o desenvolvimento de uma interface gráfica para exibição dessas transmissões. 


\section{REFERÊNCIAS}

[1] PASTOR, E.; LOPEZ, J.; ROYO, P. UAV Payload and Mission Control Hardware/Software Architecture. IEEE A\&E Systems Magazine, Catalonia, n. 7, p. 3-8, 2007.

[2] SILVA, K.L. da; MORAIS, A.S. de. Hardware para controle avançado de veículo aéreo não tripulado do tipo quadricópetro. Horizonte Científico, Uberlândia, v. 8, n. 1, 2014.

[3] FURTADO, V.H. et al., Aspectos de segurança na integração de veículos aéreos não tripulados (VANT) no espaço aéreo brasileiro. Sitraer, Rio de Janeiro, v. 7, n. 494, p. 506-517, 2008.

[4] RIBEIRO, Rômulo Eugênio. STT - Um Sistema para Telemetria e Telecomando de Aeronaves do Projeto ARARA. 2000. Dissertação (Mestrado em Ciências de Computação e Matemática Computacional) Instituto de Ciências Matemáticas e de Computação - ICMC, Universidade de São Paulo, São Paulo.

[5] 20a COMPETIÇÃO SAE BRASIL AERODESIGN, 2018, São Paulo. 139 p.

[6] JORGE, A. de C.; INAMASU, R.Y. Uso de veículos aéreos não tripulados (VANT) em Agricultura de Precisão. Embrapa Instrumentação, São Paulo, p. 109-134, 2014.

[7] RODRIGUES, Luiz Eduardo Miranda José. Fundamentos da engenharia aeronáutica com aplicações ao projeto SAE-AeroDesign: aerodinâmico e desempenho. São Paulo: edição do autor, 2014.

[8] MONK, Simon. Programação com Arduino: começando com Sketches. 12. ed. Porto Alegre: Bookman, 2013.

[9] PENGERTIAN Pesawat. [S. 1.], 2011. Disponível <http://afrizaltheaviation.blogspot.com/2011/03/pengertian-pesawat.html>. Acesso em: 03 fev. 2019.

[10] TAGLIETTI, L. et al. Modelagem do microcontrolador PIC 16F84 para projeto baseado no reuso de IPs. ResearchGate. 2019.

Disponível em: https://www.researchgate.net/publication/267830769_MODELAGEM_DO_MICROCONTROLADOR_ PIC_16F84_PARA_PROJETO_BASEADO_NO_REUSO_DE_IPS >. Acesso em: 04 fev. 2019.

[11] SANDERS, L.C.; CORADINI, W.F.Z. Sistema de aquisição de dados para um aeromodelo. The Journal of Engineering and Exact Sciences - JCEC. Viçosa, v. 3, n. 5, p. 733-735, 2017.

[12] MATOS, S.N. Tutorial para criar um sistema de aquisição de dados remoto, alimentado por células fotovoltaicas. Ouro Preto, 2015. Disponível em: http://professor.ufop.br/sites/default/files/adrielle/files/tutorial_para_sistema_telemetrico_sustentavel.pdf >. Acesso em: 03 fev. 2019.

[13] MESCOLOTI, A. et al. Desenvolvimento de um VANT autônomo utilizando hardware e materiais de baixo custo. Colloquium Exactarum. São Paulo, v. 10, n. 1, p. 41-50, 2018.

[14] RANA, M.M.; SAHABUDDIN, M.; MONDOL, S. Design and implemantation of a digital tachometer. International Journal os Scientific Engineering and Technology. Bagladesh, v. 5, n. 1, p. 85-87, 2016.

[15] TISAJ, David. Design and Construction os a Tachometer. Tese - School of Engineering and Information Technology, Murdoch University, Perth, 2014.

[16] CURRENT Sensors. [S. 1.], 6 nov. 2014. Disponível em: <https://muxindia.wordpress.com/2014/11/06/current-sensors/>. Acesso em: 02 fev. 2019.

[17] JIA, R. et al. A real-time differential anti strong interference tachometer based on Arduino. International Conference on Applied Mechanics, Electronics and Mechatronics Engineering. Guangzhou, 2016.

[18] NAKATANI, A.M.; GUIMARÃES, A.V.; NETO, V.M. Medição com sensor ultrassônico HC-SR04. International Congress on Mechanical Metrology. Gramado, v. 3. n. 10, 2014.

[19] ELECFREAKS. Datasheet: Ultrasonic Ranging Module HC-SR04.

[20] SOUSA, R.R. de S.; MARGI, C.B. Sensoriamento sem fio em aeromodelos radiocontrolados. Simpósio Brasileiro de Telecomunicações. São Paulo, v. 31, 2013.

[21] SILVA, Túlio Dapper e. Instrumentação de um veículo aéreo não tripulado para análise de sua performance. Monografia (Engenharia de Controle e Automação) - Escola de Engenharia, Universidade Federal do Rio Grande do Sul, Rio Grande do Sul, 2017.

[22] SERBEZOV, V.; DIMITROV S.; RANGELOV, K. Mobile test stand for evaluation of electric power plants for unmanned aircraft. MATEC Webof Conferences. Bulgaria, v. 10, n. 133, 2017.

[23] LAUB, Joseph et al. Telemetry systems for the Union College Aero Design Team. 2015. Disponível em: <https://cpb-us-w2.wpmucdn.com/muse.union.edu/dist/b/323/files/2015/03/Joseph-Laub-ECE-499Report.pdf>. Acesso em: 01 fev. 2019.

[24] PEBRIANTI, Dwi et al. Leader-Follower of quadrotor micro aerial vehicle. Journal of Telecommunication, Electronic and Computer Engineering. v. 10, n. 1, p. 67-73, 2018. 
[25] U-BLOX. Datasheet: NEO-6 u-blox 6 GPS Modules.

[26] DAĞ, Fatih Mehmet; TAMER, Özgür. Conceptual Design of Low Altitude Unmanned Small Hybrid Airship. International Research Journal of Electronics \& Computer Engineering, [S. 1.], 2018.

[27] FILARDI, V.L. Desenvolvimento de um sistema para navegação e telemetria de aeronaves não-tripuladas. Dissertação (Mestrado em Mecatrônica) - Departamento de Engenharia Mecânica e Departamento de Ciência da Computação, Universidade Federal da Bahia, 2006. 